\title{
Integração do MOODLE com repositórios abertos
}

\section{Liane Margarida Rockenbach Tarouco}

Professora Titular do Departamento de Estudos Especializados da Faculdade de Educação da UFRGS Diretora do Centro Interdisciplinar de Novas Tecnologias na Educação / UFRGS

Alessandra Pereira Rodrigues

Graduada em Análise de Sistemas. Mestre em Ciência da Computação (UFRGS).Doutorado em andamento em Informática na Educação (UFRGS) Professora Ensino Básico Técnico e Tecnológico

Marcelo Augusto Rauh Schmitt

Graduado em Computação.Mestre em Computação.Doutor em Informática na Educação (UFRGS).Professor Ensino Básico Técnico e Tecnológico.Coordenador de área acadêmica de Informática

Com a disseminação da Tecnologia de Informação e Comunicação no ambiente educacional, aliada à proliferação de ferramentas de autoria, a quantidade de material educacional digital sendo produzido aumentou significativamente. Tal situação acarretou a necessidade de ampliar o escopo dos repositórios digitais, com o propósito de incluir, nesses ambientes, conteúdos educacionais digitais produzidos segundo a estratégia de Objetos de Aprendizagem (OA), com vistas a favorecer a disponibilização e a reusabilidade desses conteúdos. Com esse novo escopo, esse tipo de repositório passou a ser conhecido como Repositórios de Objetos de Aprendizagem (ROA). A integração dos ROA com Ambientes Virtuais de Aprendizagem (AVA) tem o objetivo de facilitar o uso dos OA por parte dos professores, uma vez que o ambiente no qual os professores atuam mais frequentemente é o AVA. Este artigo apresenta uma estratégia para implementar a integração de ROA com AVA, exemplificando com uma implementação pautada pela estratégia proposta e que levou à integração de um ROA baseado no padrão Learning Object Metadata (LOM) com o AVA Modular Object-Oriented Dynamic Learning Environment (MOODLE). 
Palavras-chave: Repositórios digitais; Objetos de aprendizagem; Metadados; Ambientes Virtuais de Aprendizagem.

\section{MOODLE integration with open repositories}

Information and Communication Technology has been spread out through educational scenery as well as authoring tools proliferation, the amount of digital educational material being produced has meaningfully increased. This situation led to the necessity of expanding the scope of digital repositories aimed at including in these environments digital educational contents produced according to the strategy of Learning Objects (LO) in order to foster the availability and reusability from these contents. Within this new scope this type of repository has been known as Learning Object Repositories (LOR). The integration of crowns with Learning Management Systems (LMS) has as goal to facilitate the use of LO by teachers, since the environment in which teachers work more frequently is the LMS. This article presents a strategy to initiate the integration between LOR and AVA illustrating with an implementation strategy guided by the proposal and which led to the integration of an LOR based on the standard LOM with the LMS MOODLE.

Keywords: Digital repositories; Learning objects; Metadata; Learning management system.

Recebido em 03.04.2012 Aceito em 08.02.2013

\section{Introdução}

O processo de digitalização de recursos bibliográficos ocorre nas bibliotecas em todo o mundo, motivado tanto pela intenção de ampliar o acesso ao acervo mediante sua disponibilização para consulta via Internet quanto pela impossibilidade de conseguir espaço físico para armazenar a quantidade crescente de publicações produzidas.

Alguns exemplos dessa tendência podem ser encontrados nas maiores bibliotecas do planeta. A maior delas, a biblioteca do congresso americano em Washington, que armazena mais de 142 milhões de itens, sendo 32 milhões de livros e 63 milhões de manuscritos (RAYMONF, 2009). Um primeiro movimento, levou a um padrão denominado Z39.50, que foi adotado e teve muitas implementações, inclusive no Brasil 
(SCHWAN; TAROUCO, 1993). Esse padrão contempla a especificação de um protocolo de comunicação entre computadores projetados para permitir pesquisa e recuperação de informação (ROSETTO, 1997), baseada em uma arquitetura cliente/servidor. A proposta evoluiu e apresenta inúmeras implementações em software comercial e software livre (ANSI, 2003). Isso ensejou a formação de consórcios de universidades - bibliotecas universitárias que ofereciam serviços integrados aos seus usuários, que passaram a ter a possibilidade de efetuar uma consulta em qualquer um dos pontos de acesso da rede e recuperar informações bibliográficas sobre o acervo de todas as bibliotecas consorciadas. O protocolo Z39.50 permite realizar consultas que são replicadas para diversos servidores. Uma vez localizado o livro desejado (ou outro recurso bibliográfico), o mesmo pode ser solicitado mediante a opção de empréstimo entre bibliotecas, usando o protocolo Z39.50.

A formação de consórcios de bibliotecas não ocorreu no Brasil, tal como seria desejável. A alternativa mais usada no país foi o sistema COMUT do Instituto Brasileiro de Informação em Ciência e Tecnologia (IBICT) que oferece uma solução para os pesquisadores com vistas a proporcionar a obtenção de materiais que somente pudessem ser encontrados em bibliotecas remotas. O COMUT permite a obtenção de cópias de documentos técnico-científicos disponíveis nos acervos das principais bibliotecas brasileiras e em serviços de informação internacionais (IBICT, 2010).

A proliferação de sistemas de escaneamento de baixo custo levou a outra estratégia, envolvendo a digitalização de acervos e algumas bibliotecas passaram a prover serviços de cópia em meio digital, em complemento ou substituição às cópias em papel. Constata-se, atualmente, uma gradual substituição de documentos impressos por sua versão em meio digital, na medida em que seus usuários passam a acessar e manusear materiais apenas em versão digital. Assim, as bibliotecas estão gradualmente digitalizando seu acervo para automatizar - acesso aos documentos, que não tenham restrições de copyright, ampliando, assim, a oferta de serviços. Isso é especialmente importante no caso de obras raras ou antigas, que devem ter sua integridade física preservada e ser protegidas de manipulação inadequada ou excessiva. Graças a esse movimento, é possível, atualmente, ter acesso a obras que, de outro modo, dificilmente poderiam ser consultadas por um pesquisador por falta de condições de ir até a biblioteca, onde o recurso desejado está armazenado. Na Berlin State Library, fundada em 1661, parte do acervo com aproximadamente 10 milhões de itens já está digitalizada e isso torna possível a recuperação de obras produzidas no século XVI, tal como a do autor Abraham Rockenbach (1606), um professor de Matemática, Grego e Ciências Jurídicas da Universidade de Frankfurt (am Oder).

Outro fator que sustenta o uso de repositórios digitais deriva do fato de que o tipo de conteúdo sendo produzido atualmente para fins educacionais tende a utilizar multimídia. Isso torna inadequada a forma 
impressa para sua veiculação, demandando a necessidade de armazenar e dar acesso a versões digitais, contendo não apenas texto, mas, também, animações, vídeo, áudio, etc. Um exemplo desse novo tipo de conteúdo pode ser encontrado na Biblioteca do Congresso dos Estados Unidos, que armazena, atualmente, mais de 3 milhões de itens de áudio. Em 2009, essa biblioteca já continha mais de 15 milhões de itens digitais online (LOC, 2009).

No Brasil, através da iniciativa do Instituto Brasileiro de Informação em Ciência e Tecnologia (IBICT), no final de 2001, foi elaborada uma proposta para construir uma biblioteca digital brasileira. Isso levou ao projeto da Biblioteca Digital para Teses e Dissertações (BDTD) (SOUTHWICK, 2006). O estudo de viabilidade preliminar apontou duas possíveis direções para o projeto BDTD: (1) desenvolvimento de um padrão nacional de metadados para Electronic Theses and Dissertations (ETD); (2) adoção de várias soluções para integração de repositórios, como: motor de metabusca, padrão Z39.50 e protocolo Open Archive Initiative for metadata harvesting (OAI-PMH) (OAI, 2010). A estratégia selecionada foi baseada no protocolo OAI-PMH, como mecanismo de integração de repositórios de teses e dissertações. O BTDB tem sido estendido para várias universidades da América do Sul, como Argentina, Colômbia, Uruguai e Venezuela (SOUTHWICK, 2006).

Cabe salientar que a popularização de ferramentas de autoria multimídia, implementadas em software livre, também leva a um aumento considerável no volume de conteúdos em meio digital e a responsabilidade por seu armazenamento e disponibilização também começou a migrar para as bibliotecas, que passaram a ser equipadas com sistemas de armazenamento e disponibilização online de outros tipos de conteúdo, diferentes do que tradicionalmente ocorria. Isso também decorre de uma mudança de paradigma educacional que está ocorrendo no país, com a ampliação da educação a distância, que, naturalmente, trabalha com ambientes online e demanda 0 acesso aos conteúdos educacionais apropriados, com boa disponibilidade.

O armazenamento digital proporciona, além de acesso simultâneo, consulta sem deslocamento geográfico, velocidade de recuperação do conteúdo e melhores mecanismos de busca. Entretanto, esse meio acarreta alguns problemas, tais como a falta de compatibilização do formato dos conteúdos, as questões ligadas a direitos autorais, o volume dos arquivos, os mecanismos que permitam uma inspeção preliminar dos materiais para avaliar sua adequação às necessidades de um dado usuário, em um dado momento, etc. Alguns desses problemas estão sendo resolvidos com o armazenamento dos conteúdos educacionais em repositórios digitais, nos quais a catalogação dos itens é realizada com o uso de um conjunto de metadados capazes de expressar informações suficientes para permitir ao usuário selecionar previamente o que vai recuperar e inspecionar mais detalhadamente. 
Muitos repositórios passaram a ser construídos de forma independente uns dos outros, obrigando o usuário a acessar e consultar diversos sistemas isolados para conseguir encontrar os conteúdos educacionais digitais almejados para uso nas unidades de aprendizagem sendo preparadas. A falta de integração entre repositórios e, em especial, a falta de integração com ambientes virtuais de aprendizagem é um problema atual que dificulta o trabalho dos professores na preparação de aulas.

A cultura midiática nos apresenta um cenário de facilidades e eficiência na obtenção das informações, que nos causa uma impaciência quando há falta de praticidade no desenvolvimento das nossas tarefas cotidianas, como, por exemplo, a localização e veiculação de recursos educacionais digitais em AVA. Constata-se que as tecnologias digitais favoreceram a produção, localização e veiculação desses recursos, mas, quanto maior é a disponibilidade tecnológica, tanto maior é a exigência de ferramentas e sistemas que facilitem o acesso rápido e supra todas as necessidades eminentes.

O presente trabalho traz um relato de estratégias possíveis para minorar essa situação. É apresentado o cenário dos repositórios digitais voltados para conteúdos educacionais digitais (estruturados na forma de objetos de aprendizagem), bem como os problemas e soluções inerentes a essa abordagem. Um modelo para a integração dos repositórios de objetos de aprendizagem aos ambientes virtuais de aprendizagem é descrito e são analisadas e comentadas estratégias para contornar alguns dos problemas mencionados.

\section{Autoria e armazenamento de conteúdo educacional}

Com o objetivo de evitar a replicação de esforços na produção de conteúdos educacionais digitais, a comunidade internacional decidiu construí-los em pequenos segmentos orientados à reusabilidade. Esses conteúdos passaram a ser designados de objetos de aprendizagem em analogia à estratégia usada para melhorar a reusabilidade de segmentos de código de software que já era amplamente usada com bons resultados. Assim, para alcançar maior produtividade na produção de conteúdo educacional digital, os desenvolvedores passaram a estruturar o que era produzido, usando o paradigma da programação orientada a objetos, que se mostrou muito eficiente na Engenharia de Software. Através da estruturação dos conteúdos educacionais digitais em objetos de aprendizagem, foi possível melhorar sua reusabilidade e, dessa forma, melhorar a eficiência na produção de futuros conteúdos, mediante o reuso e adaptação de objetos de aprendizagem existentes.

A reusabilidade dos OA é fundamental, ao se considerarem os elevados custos de produção de conteúdos educacionais digitais. Mas, para viabilizar essa reusabilidade, é essencial dispor de sistemas nos quais os objetos de aprendizagem possam ser armazenados, catalogados de forma apropriada, com vistas a facilitar sua busca e recuperação. Um 
conjunto apropriado de metadados precisa ser associado aos objetos de aprendizagem, visando a facilitar sua localização e a análise de sua utilidade como elemento componente de novos objetos de aprendizagem sendo desenvolvidos (TAROUCO; FABRE; TAMUSIUNAS, 2003). Alguns exemplos de padrões de metadados usados atualmente em repositórios digitais são o Dublin Core (DCMI, 2011) e o Learning Object Metadata (LOM) (LOM, 2010). O primeiro foi criado para uso na categorização de conteúdo existente na Web. O segundo foi desenvolvido pelo Learning Technologies Group do Institute of Electrical and Electronics Engineers (IEEE) e adotado pelo Comitê de Padrões de Tecnologia de Aprendizagem (LTSC - Learning Technology Standards Committee) da International Standards Organization (ISO) para catalogar objetos de Aprendizagem (IEEE, 2002). Para produzir OAs que tenham maior reusabilidade, o professor autor deve procurar segmentar os conteúdos educacionais digitais em porções com granularidade apropriada. Objetos com granularidade maior apresentam menor reusabilidade. Todavia, podem ser melhor contextualizados, fornecendo condições para alavancar a aprendizagem. Objetos com granularidade mais fina possuem maior potencial de reusabilidade pela independência de contexto, mas precisam ser organizados em objetos mais complexos onde a contextualização é promovida. É importante, também, ressaltar que os objetos de aprendizagem desenvolvidos precisam estar disponíveis e facilmente acessíveis, para que possam ser encontrados e reusados. Por isso, tornase imprescindível dispor de mecanismos para localizar os objetos de aprendizagem existentes. Sistemas genéricos de busca na Internet não são suficientes, porque nem sempre tais recursos podem ser indexados, uma vez que as informações sobre seu conteúdo não podem ser derivadas de dados existentes, tais como nome de arquivo e o conteúdo, que nem sempre pode ser facilmente indexado, como é o caso de vídeos, áudio, animações, etc. Por esse motivo, são necessários o registro e indexação desses objetos em repositórios de objetos de aprendizagem.

A catalogação é baseada em metadados educacionais que descrevem várias características de um objeto de aprendizagem, com vistas a promover a disseminação de informações sobre os recursos e sua recuperação (HARMAN; KOOHANG, 2007). Caso o número de informações necessárias para cadastrar um objeto de aprendizagem em um repositório seja muito elevado, com metadados em excesso e cuja utilidade não é intuitivamente percebida pelo autor, ele tende a omitir informações no cadastramento, que acaba ficando incompleto. Os metadados básicos mais utilizados na catalogação de objetos de aprendizagem incluem: título, autor, palavras-chave, endereço do conteúdo, descrição, data, formato, linguagem, tipo de recurso educacional e público alvo, conforme levantamento de Friesen (2004). 


\section{Repositórios de objetos de aprendizagem}

Mustaro et al. (2007, p. 132) definem um repositório de objetos de aprendizagem como um catálogo digital que facilita a pesquisa por objetos de aprendizagem. Essas aplicações devem permitir o armazenamento propriamente dito, o controle de versões e de publicação, a busca dos objetos a partir de suas características, o controle de acesso e a avaliação dos objetos. Os repositórios digitais, com o objetivo de armazenar objetos de aprendizagem, diferem de outros repositórios digitais, especialmente por se tratarem de locais que reúnem recursos educacionais, os quais podem ser utilizados em diferentes contextos, editados ou agrupados a outros recursos, dando origem a um novo OA, que, por sua vez, pode ser também disponibilizado. Esta característica sugere que os recursos educacionais armazenados em um ROA sejam livres e abertos. $O$ movimento dos Recursos Educacionais Abertos (REA) pretende romper as barreiras e promover e permitir o compartilhamento livre de conteúdo tal como defendido no trabalho publicado pelo Centre for Educational Research and Innovation (CERI) da Organization for Economic Cooperation and Development (OECD) (CERI, 2007).

$\mathrm{Na}$ produção desses recursos educacionais, devem ser levadas em consideração características como a granularidade, interoperabilidade e reusabilidade, as quais possibilitarão que esses recursos sejam usados e reutilizados em diversos contextos e dispostos em diferentes ambientes de aprendizagem. Para garantir a interoperabilidade dos recursos educacionais, é recomendado que os mesmos sejam produzidos, seguindo padrões reconhecidos como LOM (LOM, 2010), Instructional Management Systems (IMS) (IMS, 2011), Sharable Content Object Reference Model (SCORM) (ADL, 2011), Dublin Core Metadata Initiative (DCMI) também referido simplesmente como Dubin Core (DCMI, 2011), entre outros, os quais permitem o cadastro de metadados que facilitam a localização do recurso educacional apropriado à situação de aprendizagem que o professor deseja construir. Os padrões citados possuem metadados diferentes e a maioria deles é oriunda do padrão LOM. Embora o padrão Dublin Core seja mais simples do que o LOM, tem sido amplamente utilizada justamente pela facilidade de implementação.

Grande parte dos repositórios digitais utiliza Content Management Systems (CMS) na sua implementação. Um CMS é uma aplicação Web que permite a criação de páginas para a $W e b$, bem como seu gerenciamento. A ideia central é permitir que usuários com pouco domínio das linguagens utilizadas na Internet, possam gerenciar uma razoável quantidade de conteúdo. Assim, programas como DSpace, Joomla, Mambo, Drupal e Plone permitem que usuários construam páginas a partir de modelos e ferramentas disponibilizados. Algumas capacidades que caracterizam um CMS são as seguintes (RODRIGUES et al., 2009): definição e alteração do layout através de modelos (templates), organização do conteúdo de forma hierárquica, definição de estruturas de menus, definição de papéis de usuários relacionados com o fluxo de publicação (leitor, autor, revisor, 
editor, administrador), adição de ferramentas de terceiros (fóruns, chats, repositórios de imagens, repositórios de arquivos) e controle de fluxo de trabalho (workflow). Algumas ferramentas que oferecem serviços de CMS foram analisadas: eGroupware da empresa Stylite AG (STYLITE, 2012), Moodle Repository Create, Upload, Tag and Embed (MrCute) 2 (KILCOYNE, 2009) e DSpace (DSPACE, 2010).

Um CMS não é um software com fins educacionais, embora possa ser utilizado para isso. Não apresenta as ferramentas necessárias para administração de cursos à distância, não é uma ferramenta de autoria para educação e tampouco é um repositório para objetos de aprendizagem. De fato, trata-se de um software utilizado por instituições para publicação de conteúdo na Internet. No entanto, a coordenação de ações utilizada para controlar a publicação de conteúdos pode ser muito útil na construção colaborativa de conteúdo educacional (RODRIGUES et al., 2009). O DSpace é um exemplo de CMS utilizado por um grande número de instituições que implementam seus repositórios digitais (DSPACE, 2011), tais como o Banco Internacional de Objetos Educacionais (BIOE) do Ministério da Educação (MEC) (AFONSO et al., 2011), o Lume da Universidade Federal do Rio Grande do Sul (UFRGS) (LUME, 2011) e o Massachusetts Institute of Technology (MIT) (MIT, 2010).

Mesmo dispondo dos recursos educacionais em acervos digitais e facilitando seu acesso por meio de consulta através dos seus metadados, que facilitam a localização dos recursos adequados, persiste o problema da diversidade de repositórios disponíveis, fazendo com que o professor necessite vasculhar em vários ambientes com interfaces distintas, até encontrar o que realmente necessita. Nesse sentido, o projeto Federação Educa Brasil (FEB) (FEB, 2010) desenvolveu uma solução que permite integrar repositórios, provendo a busca de recursos educacionais através de uma única interface. Dessa forma, o professor a partir dessa interface, realiza sua pesquisa em vários repositórios digitais, sendo mais provável a localização do recurso pretendido.

Nesse contexto e considerando que grande parte dos professores que fazem uso de recursos educacionais digitais na sua prática pedagógica utiliza ambientes virtuais de aprendizagem para compor as unidades de aprendizagem, que, por sua vez, formam o curso/disciplina, deve-se prover meios para esses recursos possam ser facilmente integrados nesses ambientes. Esse é outro problema que persiste, a incorporação desses recursos educacionais, de forma fácil e rápida, nos ambientes virtuais de aprendizagem.

\section{Modelo para integração de ROA a AVA}

A organização dos recursos educacionais disponíveis, complementada pelo planejamento de uso das ferramentas de comunicação e colaboração, com o objetivo de efetivar o processo de ensino-aprendizagem é, muitas vezes, realizada através de um AVA. Por outro lado, a busca e recuperação de recursos educacionais já 
desenvolvidos, com o objetivo de poupar tempo e reutilizar materiais de qualidade produzidos anteriormente, são realizadas em diversos ROA distintos. Devido à dissociação entre os ambientes virtuais de aprendizagem e os repositórios de objetos de aprendizagem, os profissionais responsáveis pela criação de cursos ou disciplinas em instituições ensino - geralmente professores - realizam duas tarefas intimamente relacionadas em dois ambientes distintos.

Considerando que cada ROA apresenta uma interface de navegação diferente e que a busca deve ser feita em vários repositórios, resta ao professor, que está criando uma unidade de aprendizagem, dominar uma multiplicidade de interfaces. No quadro 1 , é possível identificar a sequência de tarefas realizadas pelo usuário que utiliza os repositórios com o objetivo de buscar OA para compor as unidades de aprendizagem de uma disciplina/curso e o AVA para disponibilizá-las aos alunos. Além do domínio da interface de cada repositório consultado, a eventual cópia de um objeto de aprendizagem é feita em dois passos: download para o computador pessoal e upload para o ambiente virtual de aprendizagem.

Quadro 1 - Tarefas realizadas na criação de uma unidade de aprendizagem sem a integração do AVA com o ROA

a) acessar a interface do ROA;

b) realizar a busca por um objeto de aprendizagem;

c) encontrar o objeto de aprendizagem desejado e, então baixá-lo; senão, acessar outro ROA;

d) realizar o download do objeto de aprendizagem encontrado para o computador;

e) acessar a interface do AVA;

f) fazer o upload do objeto de aprendizagem; e

g) organizar o objeto de aprendizagem no curso.

Fonte: Elaboração própria

Para minimizar os problemas mencionados advindos da falta de integração dos ambientes ROA e AVA, decidiu-se propor e implementar uma solução que permitisse a integração desses dois tipos de sistemas de apoio à educação. O modelo proposto tem como princípio norteador a centralidade do AVA como interface para os professores de uma instituição de ensino. Esse ambiente constitui-se no ponto de entrada utilizado para busca de recursos educacionais durante o planejamento e a montagem de cursos ou disciplinas. Tal abordagem está de acordo com Fertalj, HoicBozvic, Jerkovic (2009), que sustentam que a melhor solução é aquela em que o usuário utiliza o AVA institucional para acessar o ROA. O quadro 2 apresenta a sequência de tarefas realizadas pelo usuário no modelo proposto.

Quadro 2 - Tarefas realizadas na criação de uma unidade de aprendizagem com a integração do AVA com o ROA

a) acessar interface do AVA;

b) realizar a busca por um objeto de aprendizagem nos ROA selecionados;

c) fazer o download do objeto de aprendizagem para o AVA; e

d) organizar o objeto de aprendizagem no curso.

Fonte: Elaboração própria

Perspectivas em Ciência da Informação, v.18, n.1, p.66-85, jan./mar. 2013 
De acordo com Fertalj, Hoic-Bozvic e Jerkovic (2009), embora os ambientes virtuais de aprendizagem possam exportar conteúdo para outro sistema, a colaboração com repositórios externos não é padronizada. Tal padronização é fundamental para que um ambiente virtual de aprendizagem possa interagir com um algum repositório de objetos de aprendizagem. Por essa razão, identificaram-se padrões que pudessem ser utilizados para busca e recuperação de recursos educacionais. Dois padrões constituem alternativas para recuperação de metadados associados a objetos de aprendizagem: Open Archives Initiative Protocol for Metadata Harvesting (OAI-PMH) (OAI, 2010) e Search/Retrieve via URL/Web service (SRU/W) (SRU, 2011). Após a análise dos dois protocolos, optou-se pela utilização do SRU/W, uma vez que este permite a execução de consultas complexas, fazendo com que o cliente possa solicitar ao servidor apenas os metadados dos objetos de aprendizagem que apresentem determinadas características. Enquanto o protocolo OAIPMH permite apenas a recuperação de metadados a partir de categorias pré-estabelecidas no repositório ou de timestamps, o SRU/W permite a recuperação baseada em pesquisa por termos presentes nos metadados dos objetos armazenados. Assim, o modelo proposto efetiva a integração dos AVA com os ROA, para fins de busca e recuperação de OA, através do padrão SRU/W. A Figura 1 apresenta o modelo proposto.

Figura 1 - Modelo para integração de AVA com ROA

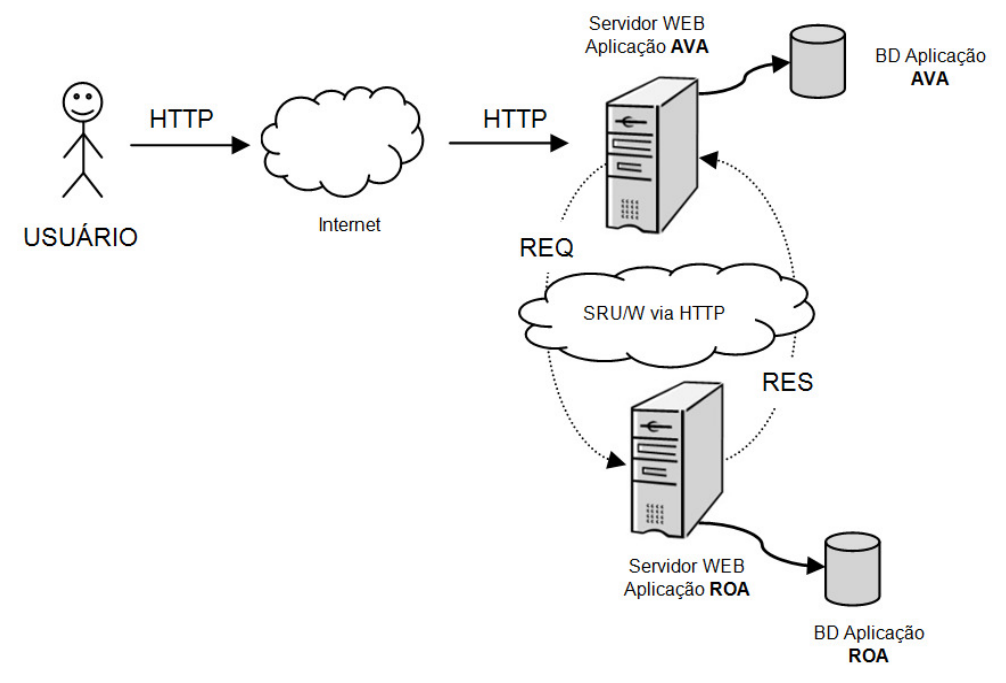

Fonte: Elaboração própria.

No modelo apresentado, o usuário utiliza apenas a interface do AVA. O módulo que provê a interface de busca e recuperação de objetos de aprendizagem no AVA comunica-se com o módulo que implementa o padrão SRU/W, a fim de realizar a consulta aos repositórios de aprendizagem e, ao receber a resposta, apresenta-as ao usuário pela mesma interface. O AVA se comunica com o ROA através do protocolo SRU/W. O módulo SRU/W do ROA traduz a consulta feita no padrão para o esquema de armazenamento local, recupera os metadados que correspondam à solicitação e retorna o resultado para o AVA. 
O processo de comunicação entre cliente e servidor é intermediado pelo protocolo de transporte Hypertext Transfer Protocol (HTTP). A Figura 2 ilustra, genericamente, o cliente e servidor e indica os passos dessa comunicação. No caso dessa implementação, o cliente refere-se ao Ambiente Virtual de Aprendizagem e o servidor refere-se ao Repositório de Objetos de Aprendizagem. O cliente, através da aplicação, envia uma consulta expressa em Contextual Query Language (CQL) para o servidor. O servidor processa os parâmetros e envia a consulta para a base de dados, a qual retorna os registros encontrados para o servidor. Os dados são formatados em XML e enviados para o cliente, usando o protocolo SRU/W:

Figura 2 - Arquitetura do Protocolo de Comunicação SRU/W

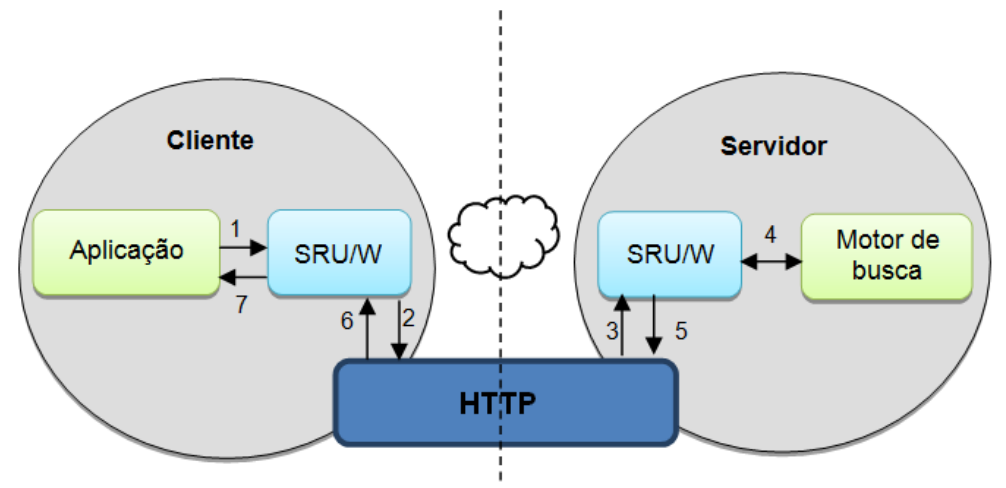

Fonte: Adaptado de Search/Retrieval via URL (2011).

\section{Implementação do modelo proposto}

A fim de testar o modelo proposto inicialmente, foi realizada a integração dos sistemas a partir da adaptação de um módulo adicional existente do MOODLE, o Moodle Repository Create Upload Tag Embed (MrCute2). Esse módulo foi desenvolvido para o MOODLE pela equipe da Worcester College of Technology, sob a coordenação de Peter Kilcoyne (KILCOYNE, 2009) e permite incorporar funcionalidades de um repositório de objetos de aprendizagem, bem como canalizar pedidos de serviço a repositórios externos como o Jorum (JISC, 2010). Decidiu-se realizar a integração entre o repositório Coletânea de Entidades de Suporte ao uso de Tecnologia na Aprendizagem versão 2 (CESTA2) e o ambiente virtual de aprendizagem MOODLE utilizado no Centro Interdisciplinar de Novas Tecnologias na Educação (CINTED). Julgou-se que tal experimento poderia trazer benefícios importantes para a comunidade de usuários, uma vez que o repositório CESTA2 é suportado por uma versão do DSpace já adaptada para utilizar o modelo de metadados LOM (TAROUCO; SCHMITT, 2010) e o AVA utilizado no CINTED é o MOODLE. Esses dois programas constituem-se, respectivamente, em um ROA e um AVA de domínio público com grande utilização no mundo inteiro.

Para que o CESTA2 pudesse comunicar-se através do padrão utilizado, foi realizada a instalação do módulo SRU/W para o DSpace, conhecido pelo nome de oclcsrwdspacelucene. Para que o MOODLE 
realizasse as consultas ao repositório, modificou-se o código de módulo denominado MrCute2. Esse código foi utilizado porque a consulta ao Jorum é realizada utilizando SRU/W (ou SRU - SRW sobre URL). A alteração realizada correspondeu ao acréscimo da alternativa de consulta ao CESTA2. Foram executadas modificações necessárias para interpretação e visualização dos metadados do CESTA2 (TAROUCO, 2010). A figura 4 mostra a tela de consulta do AVA na qual o repositório CESTA2 aparece como opção de busca. A figura 5 mostra o resultado de uma busca neste repositório através da interface do AVA. Pode-se notar que a busca foi realizada dentro do próprio ambiente virtual de aprendizagem, no repositório JORUM e no repositório CESTA2.

Figura 3 - Tela de busca do MrCute modificado, Figura 4 - Tela de resultado do MrCute modificado incluindo o CESTA2

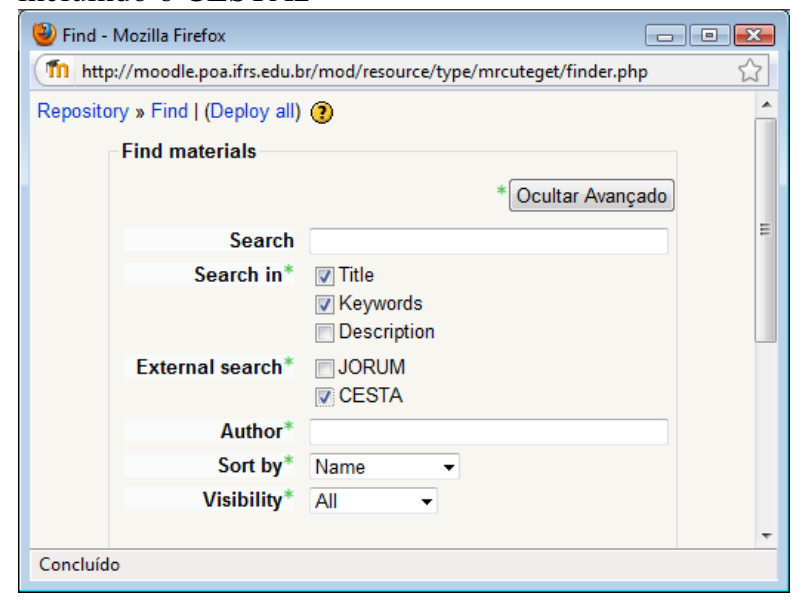

Fonte: Captura pelos autores.

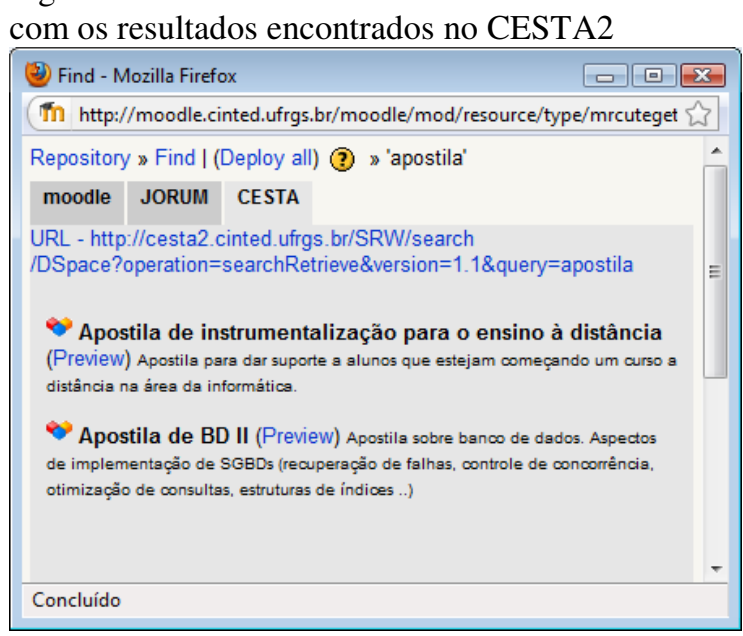

Fonte: Captura pelos autores.

O protótipo demonstrou que o protocolo SRU/W atende às necessidades de integração de ambientes virtuais de aprendizagem com repositórios de objetos de aprendizagem no que diz respeito à busca e recuperação de recursos já criados e disponíveis em repositórios. No entanto, a alteração do código permitiu somente o acesso aos metadados utilizados pelo DSpace e não se constituiu em uma solução genérica para que o MOODLE acesse qualquer repositório que compreenda o padrão SRU/W. Com o lançamento da versão 2.0 do MOODLE (DOUGIANAS, 2010), foi realizada uma análise para verificar a melhor estratégia para dar prosseguimento na implementação da integração ROA a AVA. A análise propiciou as seguintes evidências: as principais funcionalidades do módulo MrCute, como a possibilidade de armazenar OA no formato IMS em repositório local do MOODLE e recuperação de OAs contidos em um repositório externo, já estão embutidas em aplicações estruturadas na implementação padrão da versão 2 do MOODLE, não sendo necessária a instalação de nenhum módulo adicional. Auxiliando nesse processo, a versão 2 do MOODLE facilita o processo de integração com outros repositórios externos, através do fornecimento de uma Application Program Interface (API), que provê classes e métodos para facilitar a construção de novos plug-ins, utilizando o formato de classes padrão do MOODLE. Por 
esses motivos, percebeu-se que a estratégia mais interessante seria investir na implementação de um plugin para a versão 2 do MOODLE, 0 qual permitisse a conexão com repositório com as características do DSpace. Além disso, essa estratégia propiciaria desenvolver uma solução de integração mais genérica, que pudesse ser utilizada por qualquer repositório implementado, a partir do repositório DSpace ou de outro tipo de repositório que utilize o SRU (Search/Retrieve via URL) e o SRW (Search/Retrieve via Web Services) como protocolos de comunicação.

Na segunda etapa de implementação, a integração de repositórios na versão 2 do AVA MOODLE se dá através da implementação de plugins para cada repositório. Os plugins são implementados através de uma API que implementa classes, funções e métodos padrão, que permitem o desenvolvimento de novos plugins. Utilizando o mesmo protocolo de comunicação SRU/W, foi desenvolvido um plugin com base na API de Repositório fornecida pela versão 2 do MOODLE. Esses plugins são trazidos para a interface do MOODLE, através de uma única aplicação chamada Seletor de Arquivos (File Picker). Para todas as atividades e recursos disponíveis no MOODLE, nas quais é permitido anexar arquivos, inserir imagem, vídeo, adicionar arquivos, o File Picker é apresentado e, através dele, todos os repositórios integrados ao MOODLE. Todas as atividades padrão existentes no MOODLE viabilizam a chamada ao File Picker, através do editor de textos, por meio das opções de inserir imagem e inserir vídeo ou através da possibilidade de anexar arquivos por meio de algumas ferramentas como Fórum, Lição, SCORM/AICC, entre outras. A aplicação File Picker é chamada no MOODLE de duas formas distintas, permtindo que sejam trazidos arquivos ou links, o que estende a solução proposta a praticar a integração com repositórios e com referatórios ${ }^{1}$.

Um diferencial entre as duas implementações realizadas (adaptação do código do MrCute e plugin) está na característica do plugin de permitir a busca de recursos de um repositório a partir de qualquer ferramenta do MOODLE, que admita a inclusão de arquivos ou links, através da aplicação File Picker. A solução de adaptação do código MrCute restringe-se a busca de recursos através do módulo MrCute, que é apresentado como uma ferramenta do MOODLE.

\section{Resultados e discussão}

Para cada uma das implementações foram realizados estudos de caso com objetivo de verificar a funcionalidade e a usabilidade da integração com potenciais usuários. Um primeiro experimento foi realizado com professores que tiveram a oportunidade de utilizar o repositório sob duas perspectivas: não integrado e integrado ao AVA. Percebeu-se que a integração dos sistemas, especialmente do ROA ao AVA, facilita o trabalho docente, pois disponibiliza, em um único ambiente, as principais ferramentas utilizadas na construção do plano de ensino e aprendizagem, 
mitigando o tempo necessário para realização dessa tarefa (RODRIGUES; KONRATH; TAROUCO, 2010). Esse experimento propiciou aos professores uma comparação entre as duas formas de utilização do repositório, apresentadas nos quadros 1 e 2, da seção 4. A avaliação dos professores mostrou um nível de satisfação maior ao utilizar o repositório integrado ao AVA, em comparação à utilização do repositório sem a integração com o AVA (RODRIGUES; KONRATH; TAROUCO, 2010).

Para testar a segunda implementação, do DSpace ao MOODLE 2, foram realizados dois estudos de caso que envolveram usuários dos tipos aluno e professor. Esses estudos também objetivaram verificar a funcionalidade e a usabilidade, além dos possíveis benefícios da integração realizada entre AVA e ROA. Os estudos de caso compreenderam duas turmas de alunos (Turma1 $=14$ alunos e Turma2= 19 alunos) e o professor regente de cada turma. Inicialmente, um questionário de perfil foi aplicado às duas turmas de alunos e o resultado foi analisado estatisticamente, apontando que não existe diferença entre elas, isso assegura que os dois estudos de caso realizados testaram as hipóteses com amostras homogêneas da população. Por outro lado, dentro de cada amostra, verificou-se que existem diferenças nas respostas às questões propostas para examinar o perfil, no entanto, essas diferenças indicam que as condições necessárias (acesso a computador com internet, tempo, dedicação) para utilização do repositório CESTA2 integrado ao AVA MOODLE, foram favoráveis em ambas as turmas. Complementando as análises, a observação realizada permitiu verificar diferenças nas atitudes de alguns alunos, que ajudam a explicar determinados resultados encontrados na utilização da integração.

Após um semestre de atividades, em cada turma de alunos, que incluíam o uso do repositório integrado ao AVA MOODLE por alunos e professores, foi aplicado um questionário com diversas afirmativas, usando a escala Likert $^{1}$ de 5 pontos como opções de resposta, com o objetivo de verificar o nível de concordância quanto a alguns critérios de usabilidade e funcionalidade observados pelos alunos e professores, quando da utilização do plugin. Para verificar se houve diferença significativa entre as turmas, foi aplicado o teste estatístico $U$ de MannWhitney ${ }^{1}$ e, para indicar a correlação existente entre as afirmativas, utilizou-se a correlação de Spearman ${ }^{1}$.

Foram propostas afirmativas no questionário, para que os alunos avaliassem a integração quanto à facilidade, à rapidez e à satisfação em utilizar e, quanto à facilidade e à rapidez em se aprender a utilizar o repositório CESTA2, a partir do AVA MOODLE. A afirmativa mais significativa foi: "Foi fácil utilizar o repositório CESTA2 a partir do Ambiente Virtual MOODLE", no qual o valor encontrado foi inferior ao nível de significância adotado $(p=0,039585<0,05)^{1}$. Estatisticamente, pode-se afirmar, com a probabilidade de $96 \%$ de certeza, que houve diferença entre as turmas quanto à facilidade de utilização do repositório CESTA2, a partir do AVA MOODLE. Essa diferença pode ser explicada pelas formas de 
orientações dadas pelos professores das duas turmas para o uso da ferramenta, o que tornou para uma turma mais fácil do que para outra. Além disso, o perfil da turma também contribuiu para esse resultado, a Turma 2 se mostrou mais aberta, com maior entusiasmo na realização das atividades propostas com o uso do repositório CESTA2. Ambos os professores concordaram que foi possível e fácil localizar e utilizar materiais educacionais no repositório CESTA2, a partir do AVA MOODLE.

Em relação a essa mesma afirmativa, foi determinada a correlação com as demais afirmativas testadas, a fim de determinar o grau de relacionamento entre elas. Foi possível perceber que a facilidade de utilizar a integração, nas duas turmas, está diretamente relacionada à facilidade em aprender e rapidez em utilizar a ferramenta. Quando se trata da facilidade em aprender a utilizar, em relação à facilidade de utilização do repositório CESTA2, a partir do AVA MOODLE, o coeficiente mostra um grau de correlação de média a alta, respectivamente, nas Turmas 2 e 1 (Tabela 1). A afirmativa que trata da satisfação em utilizar a ferramenta de integração apresentou correlação média com a afirmativa referente à facilidade de utilizar o repositório CESTA2, a partir do AVA MOODLE, apenas para a Turma 1 ; na Turma 2, a relação foi fraca (Tabela 1). Já a afirmativa que trata a rapidez em aprender a utilizar a ferramenta de integração apresentou correlação alta com a facilidade de utilizar apenas para Turma 2; na Turma 1 a relação foi fraca (Tabela 1 ).

Tabela 1 - Correlação de afirmativas de usabilidade nas turmas

\begin{tabular}{l|c|c|c|c}
\hline \multirow{2}{*}{ Afirmativas } & \multicolumn{2}{c}{ Foi fácil utilizar o repositório CESTA2 a partir do } \\
\cline { 2 - 5 } & \multicolumn{3}{|c}{ AVA MOODLE. } \\
\cline { 2 - 5 } & $\boldsymbol{r}^{*}$ & $\boldsymbol{p}^{* *}$ & $\boldsymbol{r}^{*}$ & \multicolumn{2}{c}{ T2 } \\
\hline $\begin{array}{l}\text { Fiquei satisfeito(a) em utilizar o repositório } \\
\text { CESTA2 a partir do AVA MOODLE. }\end{array}$ & 0,571 & 0,0330 & 0,444 & 0,0566 \\
\hline $\begin{array}{l}\text { Foi fácil aprender a utilizar o repositório CESTA2 } \\
\text { a partir do AVA MOODLE. }\end{array}$ & 0,723 & 0,0035 & 0,546 & 0,0155 \\
\hline $\begin{array}{l}\text { Foi rápido aprender a utilizar o repositório } \\
\text { CESTA2 a partir do AVA MOODLE. }\end{array}$ & 0,333 & 0,2452 & 0,822 & $<.0001$ \\
\hline $\begin{array}{l}\text { Foi rápido utilizar o repositório CESTA2 a partir } \\
\text { do AVA MOODLE. }\end{array}$ & 0,626 & 0,0167 & 0,662 & 0,0002 \\
\hline
\end{tabular}

Fonte: Dados da pesquisa.

*Correlação entre as questões.

$* *$ Probabilidade de erro $(\mathrm{p} \leq 0,05)$.

Outro critério adotado para verificar a usabilidade foi a ocorrência de erros na utilização da interface de acesso ao repositório CESTA2, a partir do AVA MOODLE. Grande parte dos alunos discordou da afirmativa: "Regularmente cometo erros ao utilizar o repositório CESTA2 a partir do Ambiente Virtual MOODLE", evidenciando que não houve grandes problemas nos acessos subsequentes ao primeiro uso da interface. Os resultados estatísticos $(U=110,0000$ e $p=0,402146)$ indicaram que não houve diferença entre as turmas. 
Para testar a funcionalidade da integração do repositório CESTA2 ao AVA MOODLE, foram propostas outras afirmativas no questionário, como a possibilidade de realizar pesquisas a materiais educacionais, a partir da integração. Nessa afirmativa, 94\% dos alunos da Turma 2 concordaram que a integração permitiu a pesquisa, comparada a $79 \%$ da Turma 1 . Quanto à facilidade de realizar pesquisas geradas pela integração do repositório CESTA2 ao AVA MOODLE, somando-se os dois níveis positivos de concordância, tem-se que $89 \%$ dos alunos da Turma 2 concordaram que a integração facilitou a pesquisa a materiais educacionais adequados para a disciplina de Banco de Dados, comparada a $72 \%$ de alunos da Turma 1. Essas afirmativas apresentaram alta correlação nas duas turmas (Tabela 2).

Tabela 2 - Correlação das afirmativas de funcionalidade nas turmas

\begin{tabular}{l|c|c|c|c}
\hline \multirow{2}{*}{ Afirmativas } & \multicolumn{3}{|c}{$\begin{array}{l}\text { A integração do repositório CESTA2 ao AVA } \\
\text { MOODLE permitiu a pesquisa de materiais } \\
\text { educacionais digitais adequados na disciplina de } \\
\text { Banco de Dados. }\end{array}$} \\
\cline { 2 - 5 } & \multicolumn{2}{|c|}{ T1 } & \multicolumn{2}{|c}{ T2 } \\
\cline { 2 - 5 } & $\boldsymbol{r}^{*}$ & $\boldsymbol{p}^{* *}$ & $\boldsymbol{p}^{* *}$ \\
\hline $\begin{array}{l}\text { A integração do repositório CESTA2 ao AVA } \\
\text { MOODLE facilitou a pesquisa de materiais } \\
\text { educacionais para o estudo dos conteúdos da } \\
\text { disciplina. }\end{array}$ & 0,790 & 0,0008 & 0,753 & 0,0002 \\
\hline
\end{tabular}

Fonte: Dados da pesquisa.

*Correlação entre as questões.

**Probabilidade de erro $(\mathrm{p} \leq 0,05)$.

Isso pode ser explicado pelo fato da integração dos sistemas ter permitido a pesquisa e, assim, ela também foi facilitada, pois trouxe a possibilidade do aluno, sem sair do AVA, pesquisar materiais educacionais para o seu estudo.

Quanto à avaliação que os alunos fizeram do benefício que a integração do repositório CESTA2 ao AVA MOODLE trouxe para a sua aprendizagem, somados os percentuais positivos de concordância tem-se $71 \%$ para a Turma 1 e $79 \%$ para a Turma 2 , sendo que apenas $7 \%$ (1 aluno) da Turma 1 discordou e $21 \%$ em ambas as turmas foram indiferentes a afirmativa. 
Figura 5 - Avaliação dos benefícios da integração para a aprendizagem

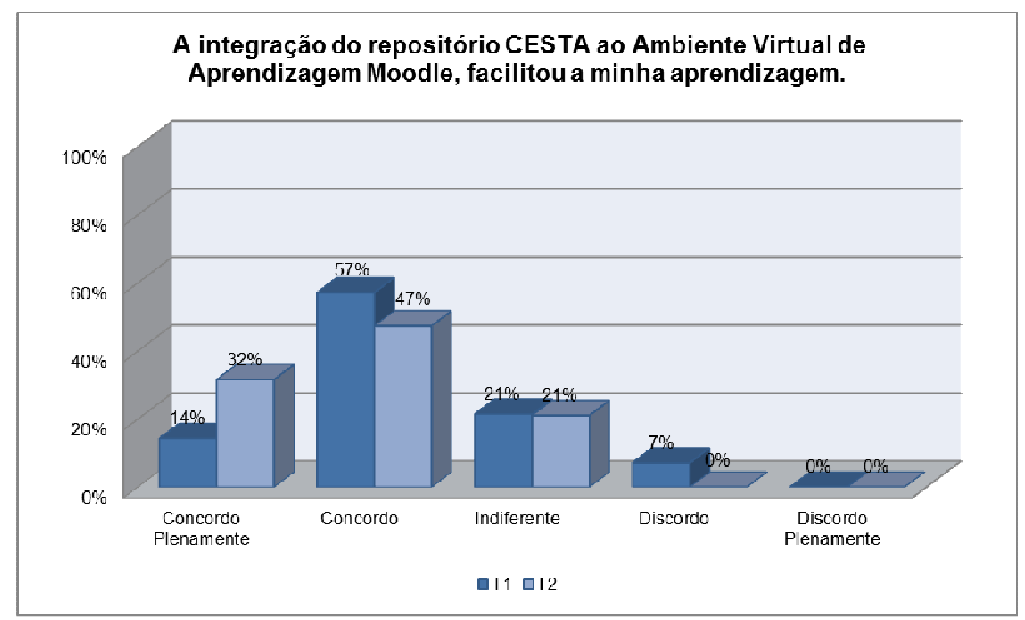

Fonte: Dados da pesquisa.

Os professores participantes do estudo confirmaram que utilizaram o repositório CESTA2 a partir do AVA MOODLE, concordaram quanto à facilidade e rapidez em utilizar, à satisfação em utilizar, à facilidade e rapidez em aprender a utilizar. Não tiveram dificuldade para retornar a utilização do repositório CESTA2, a partir do AVA MOODLE, após o primeiro uso e, discordaram quando questionados se regularmente cometiam erros ao utilizar o repositório CESTA2, a partir do AVA MOODLE. Confirmaram, também, que pesquisaram os materiais educacionais digitais referentes ao tema Banco de Dados disponíveis no repositório CESTA2 e que os consideraram relevantes para o estudo da disciplina.

Como principais resultados observados, nos estudos de casos, podese citar que a integração permitiu a pesquisa de materiais educacionais digitais adequados na disciplina de Banco de Dados, facilitou a pesquisa de materiais educacionais para o estudo dos conteúdos da disciplina, permitiu a seleção de materiais educacionais digitais adequados na disciplina de Banco de Dados, facilitou a seleção de materiais educacionais digitais adequados na disciplina de Banco de Dados, facilitou a aprendizagem do aluno e facilitou $o$ acesso a outros materiais educacionais confiáveis.

7 Considerações finais

Os testes e desenvolvimentos realizados mostram a viabilidade de se utilizar mecanismos que permitam aos docentes, usuários do MOODLE, pesquisarem repositórios externos para encontrar o conteúdo educacional apropriado para seus cursos, integrando tais objetos de aprendizagem ao seu planejamento pedagógico. O uso de repositórios externos facilita a cooperação entre instituições e o reaproveitamento de objetos educacionais existentes, potencializando o processo de criação de novos e melhores conteúdos educacionais digitais, especialmente, tendo em vista os cursos de educação à distância. A possibilidade de realizar a pesquisa e a preparação do ambiente de aprendizagem, a partir de um único 
ambiente e interface, facilitaram o trabalho do professor e permitirá ampliar a sua pesquisa, incluindo uma gama muito grande de repositórios digitais.

\section{Referências}

ADVANCED DISTRIBUTED LEARNING (ADL). Estados Unidos: [s.n.], [s.d.]. Disponível em: <http://www.adlnet.org >. Acesso em: 15 maio 2011.

AMERICAN NATIONAL STANDARDS INSTITUTE (ANSI). Information retrieval (Z39.50): application service definition and protocol specification. ANSI/NISO Z39.50-2003. Disponível em: <http://www.loc.gov/z3950/agency/Z39-50-2003.pdf > . 2003. Acesso em: 7 set. 2011.

AFONSO, M. et al. Banco Internacional de Objetos Educacionais (BIOE): tratamento da informação em um repositório educacional digital. Revista Perspectivas em Ciência da Informação, v. 16, n. 3, 2011. Disponível em: http://portaldeperiodicos.eci.ufmg.br/index.php/pci/article/view/1049/897. Acesso em: 6 fev. 2011.

DUBLIN CORE METADATA INITIATIVE (DCMI). DCMI: Home - Dublin Core Metadata Initiative. Disponível em: < http://dublincore.org/>. Acesso em: 5 ago. 2011.

DSPACE. Disponível em: <http://www.dspace.org/>. Acesso em: 1 nov. 2010.

DSPACE. Disponível em: <http://www.dspace.org/whos-using-dspace>. Acesso em: 20 set. 2011.

DOUGIANAS, M. Countdown to Moodle 2. Disponível em: <http://moodle.org/news/>. Acesso em: 11 jul. 2010.

FEDERAÇÃO EDUCA BRASIL (FEB). FEB - Federação de Repositórios Educa Brasil. Disponível em: <http://feb.ufrgs.br>. Acesso em: 3 jun. 2010.

FERTAL], K.; HOIC-BOZVIC, N.; JERKOVIC, H. Analysis of E-Leaming Repository Systems and Frameworks with Prepositions for Improvements. In: Proc. of the ITI 2009 31st Int. Conf. on Information Technology Interfaces, May 2009.

FRIESEN, N. LOM survey: final report. Dublin: [s. n.], 2004. Disponível em: <http://learningspaces.org/n/cv.html>. Acesso em: 5 maio 2010.

HARMAN, K.; KOOHANG, A. Learning objects: standards, metadata, repositories, and LCMS. California, USA: Informing Science Press, 2007.

INSTITUTO BRASILEIRO DE INFORMAÇÃO EM CIÊNCIA E TECNOLOGIA (IBICT). Programa de Comutação Bibliográfica. Disponível em: <http://comut.ibict.br/comut/>. Acesso em: 12 dez. 2010.

INSTITUTE OF ELECTRICAL AND ELECTRONICS ENGINEERS (IEEE). 1484.12.1 LOM data model standard. Copenhagen: Learning Technologies Standards Committee, 2002. 
INSTRUCTIONAL MANAGEMENT SYSTEMS (IMS). Welcome to IMS Global Learning Consortium. Disponível em: <http://www.imsglobal.org/>. Acesso em: 5 ago. 2011.

JOINT INFORMATION SYSTEM COMMITTEE (JISC). Jorum. Inglaterra. Disponível em: <http://www.jorum.ac.uk>. Acesso em: 3 mar. 2010.

KILCOYNE, P. MrCute2. Worcester College of Technology. Disponível em: <http://www.learningobjectivity.com/mrcute/>. Acesso em: 17 jun. 2009.

LIBRARY OF CONGRESS (LOC). About the library. 2007. Disponível em: <http://www.loc.gov/z3950/agency/Z39-50-2003.pdf/>. Acesso em: 18 fev. 2011.

LEARNING OBJECT METADATA (LOM). LOM. Disponível em: <http://ltsc.ieee.org/wg12/>. Acesso em: 5 ago. 2010.

LUME. Repositório Digital da Universidade Federal do Rio Grande do Sul. Disponível em: <http://www.lume.ufrgs.br/>. Acesso em: 12 dez. 2011.

MASSACHUSETTS INSTITUTE OF TECHNOLOGY (MIT). Dspace@MIT. Disponível em: <http://dspace.mit.edu/>. Acesso em: 5 ago. 2011.

MUSTARO, P. N.; SILVEIRA, I. F.; OMAR, N.; STUMP, S. M. D. Structure of storyboard for interactive learning objects development. In: KOOHANG, A.; KEITH H. (Orgs.). Learning Objects and Instructional Design. 1. ed. Santa Rosa, California: Informing Science Press, 2007. v. 3, p. 253-280.

OPEN ARCHIVES INITIATIVE (OAI). Open Archives Initiative object reuse and exchange. Disponível em: <http://www.openarchives.org/ore/>. Acesso em: 18 set. 2010.

CENTRE FOR EDUCATIONAL RESEARCH AND INNOVATION (CERI). Giving knowledge for free: the emergence of open educational resources. OECD. 2007. Disponível em: <http://www.oecd.org/dataoecd/35/7/38654317.pdf > Acesso em: 4 nov. 2010.

RAYMONF, M. How 'big' is the Library of Congress? Washington: Library of the Congress, 2009. Disponível em: <http://blogs.loc.gov/loc/2009/02/howbig-is-the-library-of-congress/>. Acesso em: 18 out. 2010.

ROCKENBACH, A. Oratio de anno epoches Christi millesimo. Frankfort on the Oder: Typis N. Voltzij, 1606. 42 p. Disponível em: $<$ http://digital.staatsbibliothek-

berlin.de/dms/werkansicht/?PPN=627401368\&PHYSID=PHYS 0001>. Acesso em: 10 out. 2011.

RODRIGUES, A. P. et al. Autoria e empacotamento de conteúdos. RENOTE, V. 7, n. 3, 2009. Disponível em: <http://seer.ufrgs.br/renote/article/view/13503>. Acesso em: 4 nov. 2010.

RODRIGUES, A. P.; KONRATH, M. L. P.; TAROUCO, L. M. R. Integração de repositórios à ambientes virtuais de aprendizagem. In: CONGRESSO LATINO-AMERICANO DE OBJETOS DE APRENDIZAGEM, 5., 2010, São Paulo. Anais... São Paulo: [s.n.], 2010. 
ROSETTO. M. Uso do protocolo Z39.50 para recuperação de informação em redes eletrônicas. Ciência da Informação, v. 26, n. 2, p. 136-139, 1997. Disponível em: <http://revista.ibict.br/index.php/ciinf/article/view/391>. Acesso em: 5 jan. 2012.

SCHWAN, R.; TAROUCO, L. Um modelo para interoperação entre sistemas de recuperação de informações. In: SIMPÓSIO BRASILEIRO DE REDES DE COMPUTADORES, 1993, Campinas. Anais... Campinas: UNICAMP, 1993. p. 684-697.

SOUTHWICK, S. B. The Brazilian electronic theses and dissertations digital library: providing open access for scholarly information. Ci. Inf., Brasília, v. 35, n. 2, p. 103-110, maio/ago. 2006.

SEARCH/RETRIEVAL VIA URL (SRU). CQL and ZeeRex (Standards, Library of Congress). Disponível em: <http://www.loc.gov/standards/sru/>. Acesso em: 25 abr. 2011.

STYLITE AG. eGroupware. Disponível em: <http://www.egroupware.org/>. Acesso em: 5 jan 2011.

TAROUCO, L.; FABRE, M.; TAMUSIUNAS, F. Reusabilidade de objetos educacionais. RENOTE, v. 1, n. 1, 2003. Disponível em: <http://seer.ufrgs.br/renote/article/viewFile/13628/7697>. Acesso em: 16 jul. 2010.

TAROUCO, L.; SCHMITT, M. Adaptação de metadados para Repositórios de Objetos de Aprendizagem. RENOTE, v. 8, n. 2, 2010. Disponível em: <http://seer.ufrgs.br/renote/article/view/15225/8988>. Acesso em: 3 ago. 2010. 